\title{
Zur Entwicklung von Aufmerksamkeit gegenüber Themen
}

Dieses Kapitel legt den zweiten von drei Teilen des theoretischen Fundaments für die Identifizierung, Beschreibung und Erklärung von Themenkarrieren in der Wissenschaft. Dabei wird das Gebiet der Wissenschaftssoziologie verlassen und stattdessen auf Ansätze der Medien- und Kommunikationswissenschaften sowie der Politikwissenschaft fokussiert. Dies hat den Hintergrund, dass letztgenannte Disziplinen die Frage nach der Entstehung von Themen (im Gegensatz zur Wissenschaftssoziologie) bereits seit Jahrzehnten verfolgen (allerdings nicht im Feld der Wissenschaft). Ziel des Kapitels ist es also, Konzepte, Merkmale und Mechanismen der Themenentstehung zusammenzutragen, deren Übertragbarkeit auf die Wissenschaft in den folgenden Kapiteln dieser Arbeit geprüft werden kann. Dies fängt bei der Klärung grundlegender Begriffe wie Thema oder Themenkarriere an und hört bei der Evaluation unterschiedlicher Ansätze der Agenda Setting-Forschung auf. Auf diese Weise sollen die Wissenschaftssoziologie und die Planungswissenschaft durch die Forschungstraditionen der Medienund Kommunikationswissenschaften sowie der Politikwissenschaft befruchtet werden.

Wie sich zeigen wird, sind die Rahmenbedingungen der Themenentstehung in den Medien, der Politik oder der allgemeinen gesellschaftlichen Öffentlichkeit andere als jene in der Wissenschaft. Eine 1:1-Übertragung hier identifizierter Theorien und Modelle auf die Wissenschaft ist schon deshalb nicht vorgesehen. Stattdessen sollen etablierte Erkenntnisse anderer Disziplinen gesammelt werden, die unter Berücksichtigung der besonderen Strukturen der Wissenschaft für potenziell erklärungsrelevant im Rahmen der Fragestellung eingestuft werden. Diese theoretische Grundlage ermöglicht schließlich die informierte Justierung und Fokussierung des analytischen Rahmens dieser Arbeit sowie der empirischen Analyse der planungswissenschaftlichen Thematisierungsprozesse. Dabei

A. Gravert, Themenkarrieren in der Wissenschaft, 
werden zum einen zentrale Begriffe der Themenentstehung eingeführt, die in der Wissenschaftssoziologie bislang nicht konzeptualisiert wurden, die aber für die empirische Analyse der Themenkarrieren in der Planungswissenschaft benötigt werden. Zum anderen wird auf einige zentrale Konzepte in Kapitel 9 und 10 rekurriert, um ihre Eignung für die Beschreibung und Erklärung der Entstehung von Themen in der Wissenschaft abzuwägen.

Zunächst werden in Abschnitt 3.1 Konzeptualisierungen der Begriffe Thema, Öffentlichkeit und Aufmerksamkeit diskutiert, die bei der Festlegung des analytischen Rahmens aufgegriffen und zu einem eigenen Verständnis zentraler Kategorien verdichtet werden (siehe Kp. 5.1). In den darauf folgenden Abschnitten werden unterschiedliche Forschungstraditionen zur Beschreibung und Erklärung der Themenentstehung erläutert. Hierzu gehören zunächst die auf Luhmann und Downs zurückgehenden Themenzyklusmodelle (Kp. 3.2). Daran anschließend werden Agenda Setting-Ansätze aus den Medien- und Kommunikationswissenschaften diskutiert, die insbesondere auf die Beeinflussung der öffentlichen Meinung durch die Salienz und das Framing von Themen fokussieren (Kp. 3.3). Das letzte Teilkapitel behandelt politikwissenschaftliche Ansätze des Agenda Setting, die die hohe Bedeutung der öffentlichen Problematisierung von Themen für das Zustandekommen politischer Entscheidungen betonen und auf die Identifizierung von Mechanismen des Setzens und Blockierens von Themen durch rivalisierende Gruppen abzielen (Kp. 3.4).

\subsection{Thema und öffentliche Aufmerksamkeit: Eine begriffsdefinitorische Auseinandersetzung}

Die Begriffe Thema und Aufmerksamkeit stellen die zentralen Untersuchungskategorien dieser Arbeit dar und bedürfen daher einer nachvollziehbaren Abgrenzung, Definition und Operationalisierung. Insbesondere in der aus der Theorie abgeleiteten empirischen Übersetzung beider Konzepte wird der Forschungsgegenstand erst konstruiert, sodass hierin ein Schlüsselmoment für die Verknüpfung von Theorie und Empirie, für die Erhebung und Auswertung der Daten und folglich für die Ergebnisse dieser Arbeit zu sehen ist. Das Feld der Wissenschaftssoziologie kann dabei nicht als Bezugsquelle dienen, da die Begriffe hier bislang nicht konzeptualisiert wurden. Es wird daher auf die Medien- und Kommunikationswissenschaften zurückgegriffen, da hier die Begriffe originär verortet sind und bereits im Zentrum vieler empirischer Analysen stehen. 
Der Begriff Thema findet je nach Anwendungsbereich unterschiedliche Verwendung, wird aber selten explizit definiert und operationalisiert. In angelsächsisch geprägten Diskursen wird Thema häufig synonym mit dem englischsprachigen issue behandelt, was wiederum zusätzliche Facetten eröffnet (s.u.). Grundlegende Quellen für die Konzeptualisierung von Thema in diesem Vorhaben sind vor allem die Theorie des kommunikativen Handelns nach Habermas sowie die Systemtheorie nach Luhmann, die beide - wie die vorliegende Arbeit - Akteur*innen und Kommunikation in den Vordergrund stellen. Zusätzlich werden Konzeptionen aus Agenda Setting- bzw. Themenzyklus-Forschungen vorgestellt, die aus den Medien- und Kommunikationswissenschaften sowie aus der Politikwissenschaft stammen.

Jürgen Habermas umreißt den Themenbegriff im Rahmen seiner kommunikationstheoretischen Konzeption der „Lebenswelt“ (Habermas 1981b: 173-293). In Abgrenzung zu phänomenologischen, subjektiven Deutungen, legt Habermas dabei ein dialogisches, intersubjektives Verständnis der Lebenswelt vor. Die Lebenswelt baut sich aus implizitem Wissen und diffusen Überzeugungen auf, die den Kommunikationsteilnehmer*innen als gemeinsamer Hintergrund ihrer Auseinandersetzung dienen (Habermas 1984: 590-591). Sie markiert den Bereich potenzieller Relevanzen der möglichen Verständigung und legt fest, was grundsätzlich „thematisierbar" ist und was nicht. Im Hinblick auf das Thema hat die Lebenswelt folglich eine kontextbildende Funktion. Das in Frage stehende Thema grenzt einen Relevanzbereich ab, der den Vordergrund einer Kommunikation bildet (ebd.). Es schränkt den Bereich in einer Kommunikation zulässiger Bestandteile der Lebenswelt ein. Nur dieser Ausschnitt ,passt ‘ dann zum Thema, ist also im Kontext der Kommunikation ,thematisierungsfähig“ (Kirsch und Weber 1999: 16). Habermas (1981a: 194) erläutert:

\begin{abstract}
„Ein Thema kommt im Zusammenhang mit Interessen und Handlungszielen (mindestens) eines Beteiligten auf; es umschreibt den Relevanzbereich der thematisierungsfähigen Situationsbestandteile und wird durch die Pläne akzentuiert, die die Beteiligten auf der Grundlage der Situationsdeutung fassen, um ihre jeweiligen Zwecke zu verwirklichen. “
\end{abstract}

Nach Habermas sind Themen also an die Beteiligten gebunden und nicht personenunabhängig zu denken. Die Existenz eines Themas ist von dem gemeinsamen Wissen über die Inhalte, die ein Thema repräsentiert, abhängig (Gerhardt 2001: 86). Themen sind darüber hinaus mit Interessen verknüpft und besitzen damit eine immanente politische Dimension (Kirsch und Weber 1999: 15). Indem die Beteiligten auf Basis ihrer jeweiligen Pläne Beiträge in die Auseinandersetzung 
einspeisen, erfährt das Thema im Rahmen der Kommunikation eine permanente Verschiebung bzw. Fortentwicklung (ebd.: 16).

In der Systemtheorie nach Niklas Luhmann nehmen Themen eine vergleichbare Funktion ein. Sie werden verstanden als „mehr oder weniger unbestimmte und entwicklungsfähige Sinnkomplexe“ (Luhmann 1970: 7). Sie liegen „als Struktur jeder Kommunikation zugrunde, die als Interaktion zwischen mehreren Partnern geführt wird“ (ebd.: 7-8). Die Frage, wie verständliche, das heißt „nichtbeliebige" Kommunikation ermöglicht wird, führt zur Differenz von Themen und Beiträgen:

\begin{abstract}
„Kommunikationszusammenhänge müssen durch Themen geordnet werden, auf die sich Beiträge zum Thema beziehen können. Themen überdauern Beiträge, sie fassen verschiedene Beiträge zu einem länger dauernden, kurzfristigen oder auch langfristigen Sinnzusammenhang zusammen. " (Luhmann 1991: 213)

„Auf der Ebene von Themen lassen sich deshalb Sinnbezüge aktualisieren, die an der Einzelkommunikation kaum sichtbar zu machen wären. “(Luhmann 1991: 216)
\end{abstract}

Während Themen also einen übergreifenden Kontext zur Einordnung von Kommunikationen darstellen, sind Beiträge die darin einzuordnenden Einzelkommunikationen.

Gemäß Luhmann (1991: 216) dienen Themen als „sachlich-zeitlich-soziale Strukturen des Kommunikationsprozesses". Die sachliche Strukturierung eines Themas zielt darauf ab, dass gemeinsame Sinnkomplexe bei den Individuen erzeugt werden, die das Aneinandervorbeireden verhindern (Gerhardt 2001: 86). Claas (2015: 51) definiert Themen dementsprechend als „Erwartungsstrukturen auf emergenter Ebene, durch welche die unendliche Menge an Möglichkeiten dessen, was man sagen könnte, im Vorfeld schon auf ein überschaubares Maß reduziert wird". Aus dieser Erwartungserwartung - also der Erwartung, die sich auf die Erwartung des Gegenübers bezüglich passender Beiträge bezieht - ergeben sich „Thematisierungsschwellen“, denn nicht alles kann mehr gesagt werden, sondern nur noch das, was als passend eingeschätzt wird (Luhmann 1991: 214). Die Strukturierung durch Themen besitzt eine zeitliche Komponente, denn Themen haben eine „Lebensgeschichte“ und entwickeln sich mit jedem neuen Beitrag fort (Luhmann 1970: 14). Ein Beitrag, der zu Beginn einer Themenkarriere für sinnvoll erachtet wird, kann zu einem späteren Zeitpunkt als unpassend abgelehnt werden oder umgekehrt. Themen selbst können ,alt oder neu, schon langweilig oder noch interessant, und all das für verschiedene Teilnehmer in verschiedener Weise" sein (Luhmann 1991: 214-215). Ein Thema strukturiert den Kommunikationsprozess sozial, indem es reguliert, ,wer was beitragen kann“ (Luhmann 
1991: 213). Indem in Abhängigkeit von einem Thema nur bestimmte Beiträge als zulässig gelten, ,diskriminieren“ Themen Beiträge und damit auch die potenziell an der Kommunikation Beteiligten (ebd.:213): Nicht jede Person kann zu einem bestimmten Thema einen adäquaten Beitrag leisten, genauso wenig wie eine bestimmte Person nicht zu jedem Thema adäquat beitragen kann. Zudem können Themen Teilnehmer*innen zusammenführen, indem sie eine (thematische) Verbindung unter ihnen herstellen, und sie können Teilnehmer*innen binden, indem sie Erwartungen bezüglich zukünftiger Kommunikationen schaffen (ebd.: 213-215).

Zusätzlich zu diesen drei einander bedingenden Dimensionen der Themenstrukturierung nach Luhmann - inhaltlich, zeitlich, sozial - lässt sich eine vierte, politische Dimension konzeptualisieren (Gerhardt 2001: 87). Schließlich ist die Entwicklung eines Themas gemäß Luhmann (2005a: 169) mit unterschiedlichen Interessen verbunden, denn es gehört „[...] zum politischen Geschick, zu spüren, in welcher Phase einer Themenkarriere man Themen aufgreift, sich von ihnen promovieren läßt, als Trittbrettfahrer von ihnen profitiert oder besser von ihnen läßt“. Mit Blick auf die Planungswissenschaft und das mit ihr korrespondierende Handlungsfeld der Raumplanung lässt sich auch eine räumliche Dimension hinzufügen. So hängt es in der Raumplanung mitunter von lokal spezifischen topologischen und institutionellen Begebenheiten ab, ob und in welcher Form ein Thema aufgegriffen wird, wie sich bspw. anhand der Analyse lokal verhandelter Wissensarten zeigen lässt (vgl. Reuter und Jessen 2019; Zimmermann 2010).

Von diesen soziologischen Thema-Konzeptionen nach Luhmann und Habermas abzugrenzen ist das Begriffsverständnis der Agenda Setting- und Themenzyklusforschung ${ }^{1}$. In der überwiegend englischsprachigen Forschungsliteratur kommt der begrifflichen und empirischen Bestimmung des Themas bzw. des issue eine Schlüsselrolle $\mathrm{zu}$, schließlich bildet es häufig die grundlegende Untersuchungseinheit (Top 2006: 76). Allerdings hat die Literatur eine Vielzahl unterschiedlicher Definitionen des issue-Begriffs hervorgebracht. Nicht zu Unrecht merken Lang und Lang (1981: 450) daher an:

„What is an issue? Without a clear definition, the concept of agenda-setting becomes so all-embracing as to be rendered practically meaningless. “

Trotz der Bandbreite der Definitionen von issue zeigt sich ein grundlegender Unterschied zu dem Themenbegriff, wie er in der Soziologie verstanden wird. Er ergibt sich aus dem unterschiedlichen Forschungsgegenstand: Während in der

\footnotetext{
${ }^{1}$ Luhmanns Themenkarriere (siehe Kp. 3.2) bildet hier eine Ausnahme.
} 
Soziologie die Strukturierung der Kommunikation zwischen Personen im Fokus steht, ist es in der Agenda Setting-Forschung die gegenseitige Einflussnahme durch das Setzen und Rahmen von Themen bzw. Problemen insbesondere in und zwischen den Bereichen Politik, Medien und Öffentlichkeit. Damit einher geht die Betonung des Konflikthaften, die bspw. in der weit verbreiteten Definition von Cobb und Elder (1972: 82) zum Ausdruck kommt:

„An issue is a conflict between two or more identifiable groups over procedural or substantive matters relating to the distribution of positions or resources. "

Der Unterschied äußert sich auch in der unterschiedlichen Denotation des Begriffs Thema gegenüber dem englischen Pendant issue (Eichhorn 2005: 8). So würde das Thema Klimawandel in der politischen oder öffentlichen Diskussion als issue bezeichnet werden, während es als Bestandteil eines wissenschaftlichen Vortrags eher mit Subject oder Topic übersetzt würde. ${ }^{2}$ Eine nähere Entsprechung des Begriffs issue im Deutschen wäre Streitfrage, öffentliches Anliegen oder Problem. In der deutschsprachigen Agenda Setting-Literatur sind insbesondere die Begriffe Thema, issue oder Streitfrage in Verwendung.

Auch die unterschiedlichen Konzeptionen von issue bieten einige Anknüpfungspunkte für diese Arbeit. Ein issue ist immer auf die öffentliche, nicht die private Sphäre bezogen. Ein Bezug auf Einzelpersonen ist daher nur vor dem Hintergrund ihrer öffentlich relevanten Rollen zulässig (Eichhorn 2005: 8-9). Ein issue umfasst immer ein oder mehrere Ereignisse, die dessen Höhepunkte und markante Zäsuren darstellen (Schulz 1976: 115). Ereignisse werden hier als diskrete, das heißt räumlich und zeitlich abgrenzbare Geschehnisse verstanden, zu denen bspw. auch mündliche Äußerungen gezählt werden können (Waldherr 2012: 12). Dieselben Ereignisse bzw. dieselben Objekte der Berichterstattung lassen sich jedoch ganz unterschiedlichen issues zuordnen, sodass sich „quasi-hierarchische Netzwerke“ ergeben (Eichhorn 2005: 9). Dabei bezieht sich die Hierarchie auf unterschiedliche Abstraktionsniveaus und meint keine eindeutige Zuordnung von unter- zu übergeordneten Themen (Leiner 2016: 9). Issues bringen also verschiedene Sub-issues bzw. untergeordnete Themen in einen Zusammenhang, sind aber selbst Teil mindestens eines weiteren, übergeordneten issues (Eichhorn 2005: 9). Der inhaltliche Gehalt eines Themas ergibt sich dann aus der Gesamtheit der verbundenen Objekte (bspw. Ereignisse oder Themen), wobei nicht jedes Objekt für ein Thema gleichermaßen bedeutsam

\footnotetext{
${ }^{2}$ Umgekehrt umfasst auch der Begriff, issue ' Denotationen, die nicht mit dem Begriff ,Thema ' abgedeckt sind (bspw. ,Ausgabe'), die allerdings im Kontext der Fragestellung keine Bedeutung haben.
} 
ist (Leiner 2016: 6). In Agenda Setting-Studien kommt der Bestimmung des Abstraktionsniveaus - bzw. der „Kategorienbreite“ (Eichhorn 2005), des „Detailgrads“ (Blumhoff und Seiffert 2014) oder des „Auflösungsgrads“ (Rössler 2006) - eines Themas eine wichtige Rolle zu. Wie konkret oder abstrakt ein Thema gefasst wird, entscheidet schließlich unter anderem darüber, welche Relevanz es im öffentlichen Diskurs entfaltet und welche Prozesse der Genese und Veränderung des Themas durch die*den Forschende*n in den Blick zu nehmen sind (Eichhorn 2005: 9).

Mit den Begriffen issue, Thema und Themenkarriere sind die Begriffe Öffentlichkeit und Aufmerksamkeit eng verknüpft. Öffentliche Aufmerksamkeit ist immer auf Themen gerichtet, Themen wiederum existieren nur, wenn öffentlich über sie kommuniziert wird und Themenkarrieren beschreiben den Verlauf öffentlicher Aufmerksamkeit gegenüber Themen (Newig 2004: 152-153).

In der kognitiven Psychologie wird Aufmerksamkeit beschrieben als die bewusste oder unbewusste Allokation knapper kognitiver Ressourcen auf einen diskreten Informationsgegenstand, bei gleichzeitiger Ausblendung anderer verfügbarer oder wahrnehmbarer Informationen (Anderson 2009: 63-91). Mit Blick auf die Fragestellung wird in dieser Arbeit die Aufmerksamkeit einer Öffentlichkeit betrachtet. Öffentlichkeit wird allgemein als Kommunikationssystem verstanden, in dem Akteur*innen sich in verschiedenen Foren und Medien über verschiedene Themen verständigen (Gerhardt 1998). Heruntergebrochen auf einzelne Kommunikationen, lässt sich Öffentlichkeit als die Gesamtheit aller potenziell an der Kommunikation beteiligten Akteur*innen betrachten. Häufig wird die Öffentlichkeit auch von den Medien bzw. der Politik abgegrenzt, um die Wechselbeziehungen zwischen den Systemen (bspw. Medien<->Publikum oder Politik<->Wähler) herauszustellen (bspw. Newig 2004).

Im Fokus der Theorien um öffentliche Aufmerksamkeit stehen folglich nicht individualpsychologische und kognitive Phänomene, sondern zwischenmenschliche, soziale Prozesse der Aufmerksamkeitsentstehung auf der Meso- und Makroebene. Ebenfalls ist das Konzept gegenüber dem Begriff der öffentlichen Meinung abzugrenzen, denn öffentliche Aufmerksamkeit bezieht sich nicht darauf, was Akteur*innen über ein Thema denken, sondern darauf, über welches Thema sie sich Gedanken machen. Während sich die öffentliche Aufmerksamkeit schnell auf ein neues Thema richten (und von einem anderen abwenden) kann, wandeln sich die Meinungen und Überzeugungen bezüglich eines Themas in der Regel nur langsam, wobei Luhmanns Konzeption der öffentlichen Meinung hier eine Ausnahme darstellt (Newig 2004: 154; Luhmann 1970).

Im Rahmen der Themenzyklus- und Agenda Setting-Forschung wird öffentliche Aufmerksamkeit dementsprechend verstanden als die Fokussierung knapper 
Ressourcen - Zeit und weiterer - von Interessengruppen, den Medien, der Politik oder der gesamten Gesellschaft darauf, Informationen zu einem bestimmtem öffentlich diskutierten Thema zu prozessieren (Ripberger 2011: 252; Ellis et al. 2011: 6; Newig 2004: 153). Hinzuzufügen ist dieser Definition der Aspekt der Kommunikation über die Informationen. Ohne diese bleibt die Aufmerksamkeit einer Vielzahl von Individuen nicht nur unerkannt, sie lässt sich auch nicht als öffentlich bezeichnen, denn Kommunikation ist das grundlegende Prinzip des Öffentlichkeitskonzeptes (Newig 2004: 155).

Über einen Zeitraum betrachtet, lässt sich öffentliche Aufmerksamkeit gegenüber einem Thema als Verlauf der Intensität verstehen, mit der die Ressourcen der Öffentlichkeit auf eben dieses Thema verwendet werden. Weil ein unerschöpflicher Fundus an Themen um ein weitestgehend gleichbleibendes Maß an öffentlicher Aufmerksamkeit konkurriert, ist öffentliche Aufmerksamkeit ein knappes Gut (Newig 2004: 153-154). Das Ausmaß, mit dem eine Öffentlichkeit ihre Ressourcen auf ein Thema konzentriert und darüber kommuniziert, lässt sich empirisch näherungsweise quantifizieren, etwa durch die Häufigkeit der Thematisierung in politischen (bspw. in Sitzungsprotokollen) oder medialen (bspw. in Tageszeitungen) Diskursen, wobei hier nicht die (schwer zu ermittelnde) kognitive Beschäftigung der Öffentlichkeit mit einem Thema, sondern nur die Kommunikation darüber als Indikator herangezogen wird.

\section{Schlussfolgerungen}

Auf der Grundlage der hier dargestellten Aufschlüsselung medien- und kommunikationswissenschaftlicher Konzeptualisierungen von Thema/issue und öffentlicher Aufmerksamkeit soll die Entwicklung eines eigenen Verständnisses erfolgen. Dabei werden die Konzepte erstmals auf das System der Wissenschaft angewendet, was ein umfassendes Verständnis von dessen Strukturen und Mechanismen (siehe Kp. 4) voraussetzt. Die - wissenschaftssoziologisch informierte - Ableitung einer eigenen Konzeptualisierung wird daher nicht an dieser Stelle vorgenommen, sondern im Rahmen der Forschungsheuristik in Abschnitt 5.1. Die Operationalisierung der Konzepte erfolgt anschließend im Rahmen der Methodik (siehe Kp. 6.2) und die Anwendung auf das Untersuchungsfeld (Planungswissenschaft) in den empirischen Kapiteln (siehe Kp. 7 und 8).

Eine im Rahmen der Forschungsheuristik dieser Arbeit einzuführende Konzeption für die Analyse von Themen bzw. issues in der Wissenschaft kann von beiden oben angeführten Traditionen - Soziologie und Agenda Setting - profitieren. Zum einen bietet die Soziologie eine tiefergehende Analyse der Funktion eines Themas als Strukturierungselement von Kommunikationen. Vor dem Hintergrund der 
in dieser Arbeit eingenommenen Perspektive ist es wichtig zu betonen, dass Themen nicht allein inhaltlich strukturiert sind, sondern dass sie auch eine zeitliche, soziale, politische und räumliche Dimension aufweisen: Themen bestimmen einen Sinnkomplex (inhaltlich), der einem permanenten Wandel unterliegt (zeitlich), zu dem nicht alle Akteur*innen einen gleichermaßen qualifizierten Beitrag leisten können (sozial), dessen Verhandlung mit Interessen verbunden und dementsprechend umkämpft ist (politisch) und der lokalspezifische Konnotationen, Verläufe und Interessen aufweist (räumlich). Für die Berücksichtigung dieser Dimensionen im Rahmen der Empirie ist wiederum die Agenda Setting-Forschung aufschlussreich, die die Bedeutung einer nachvollziehbaren und bedachten Operationalisierung hervorhebt. Als Begriff wird im empirischen Teil der Arbeit Thema gegenüber issue oder Streitfrage vorgezogen, da es in der deutschsprachigen Planungswissenschaft am ehesten ein intersubjektiv geteiltes Verständnis dessen vermittelt, worauf diese Arbeit abzielt. In den folgenden Teilkapiteln (3.2 bis 3.4) werden jedoch weiterhin auch die Begriffe issue und Streitfrage verwendet, um die im Rahmen des jeweils geschilderten Modells eingesetzte Begrifflichkeit wiederzugeben.

\subsection{Themenzyklusmodelle}

In diesem Kapitel werden Modelle zur Beschreibung und Erklärung des zyklischen Verlaufs der öffentlichen Aufmerksamkeit gegenüber Themen bzw. issues diskutiert. Diese primär in den Medien- und Kommunikationswissenschaften eingesetzten Modelle gehen von der Beobachtung aus, Politik, Medien oder Öffentlichkeit würden bestimmten Streitfragen temporär mehr Aufmerksamkeit beimessen als anderen. Sie systematisieren den Themenverlauf auf der Basis quantitativer Daten anhand von Phasen mit dem Ziel, die jeweiligen Charakteristika jenseits konkreter Fallstudien verallgemeinern zu können. Im Gegensatz zu Agenda Setting-Ansätzen, die auch systemübergreifende Kommunikationen und wechseldynamische Perspektiven ins Auge fassen, konzentrieren sich Themenzyklusmodelle auf den Themenverlauf innerhalb einer Arena (Waldherr 2008b: 301). Grundlegend für die Themenzyklusforschung und bis heute relevant sind die unabhängig voneinander entwickelten Modelle von Niklas Luhmann (1970, 1996) und Anthony Downs (1972).

Luhmann entwickelte sein Konzept der Themenkarriere aufbauend auf verhaltens- und systemtheoretischen Ansätzen und wendete das Modell zunächst auf das politische System (Luhmann 1970) und später auf das System der 
Massenmedien (Luhmann 1996) an. Dabei ist das Thema nicht unbedingt Eigenprodukt des untersuchten Systems, wird aber durch das System aufgegriffen und hier einer Karriere ausgesetzt, die sich nicht mehr durch ursprüngliche, systemexterne „Befunde“ erklären lässt (ebd.: 28). Luhmann beobachtet, dass Themen im strukturellen Rahmen des politischen Systems nicht beliebig erzeugt werden können,

„[...] sondern eine Art Lebensgeschichte haben, die, wie das Leben selbst, verschiedene Wege gehen und vorzeitig abgebrochen werden kann, gleichwohl aber nach typischen Phasen geordnet ist. In den einzelnen Phasen der Karriere eines Themas stehen jeweils bestimmte Möglichkeiten offen, sind jeweils bestimmte Probleme zu lösen, bestimmte Teilnahmevoraussetzungen zu erfüllen, und daraus ergeben sich strukturierte Handlungschancen für diejenigen, die das Thema bewegen, es fördern, bremsen, blockieren oder auf bestimmte Bahnen leiten möchten. “ (Luhmann 1970: 14)

Grundlegend für Luhmanns Modell ist das Verständnis von Aufmerksamkeit als knappes Gut. So können gesellschaftliche Teilsysteme nur eine begrenzte Anzahl von Themen gleichzeitig behandeln und müssen daher fortlaufend eine Auswahl treffen. Alte Themen müssen ,abgefertigt“ werden, um Raum für neue Themen zu gewinnen (Luhmann 1970: 15). Im Ergebnis erhalten bestimmte Themen für einen gewissen Zeitraum erhöhte Aufmerksamkeit. Sie erfahren eine Themenkarriere, die typischerweise die folgenden vier Phasen umfasst:

1. Latente Phase: Das Thema ist Eingeweihten und Interessierten bereits bekannt, allerdings ist noch nicht klar, ob, wann und in welcher Form es von Politiker*innen aufgegriffen werden wird. Noch gibt es keinen Zeit- bzw. Handlungsdruck. Häufig ,vegetieren Themen in dieser Vorform lange dahin“, bis sie die Kraft für eine Karriere gesammelt haben und die richtige Zeit für einen Durchbruch bzw. die öffentliche Thematisierung gekommen ist. Manche Themen kommen nie über die Latente Phase hinaus (Luhmann 1970: 14).

2. Durchbruchphase: Es finden sich mutige Akteur*innen zusammen, denen die Kreation des Themas gelingt, die es also rahmen und verbreiten. Sie setzen auf das Thema und widmen ihm Zeit, Ressourcen und Kontakte. Gemäß Luhmann (1970: 14) sind es oft Außenseiter, ,die nur an diesem einen Thema interessiert sind und politisch nicht viel zu verlieren haben “. Ebenso nehmen oft Neulinge zentrale Positionen ein, ,die auf dem Thema reitend eine eigene Karriere beginnen “. Unter Einsatz von Ressourcen, aber auch mit Glück und Geschick, erreichen sie, dass das Thema nicht mehr nur die Themenpromotor*innen und Interessierten beschäftigt, sondern auch auf die Agenda derer gelangt, die über wechselnde Themen entscheiden oder berichten. Allerdings 
können einige Eliten das Thema auch in dieser Phase noch ignorieren, blockieren oder auf die „Seitengleise unpolitischer Angelegenheiten“ schieben (Pfetsch 1994: 14).

3. Modephase: Es gelingt, das Thema nicht nur als wichtig und dringlich, sondern auch als politisch lösbar in der öffentlichen Auseinandersetzung zu verankern (Pfetsch 1994: 14). Es gewinnt an öffentlicher Resonanz und übernimmt seine Funktion als Strukturierungselement im Prozess öffentlicher Kommunikation (Rössler 2015: 463-465). Streitbar sind einzelne Meinungen und Entscheidungen, das strukturierende Thema selbst jedoch entzieht sich durch Selbstverständlichkeit der Disposition. Die Gegner müssen sich auf einzelne Vorbehalte, Verzögerungstaktiken oder bedingte Anerkennung zurückziehen. Die Förderinnen und Förderer müssen jetzt versuchen, es zu institutionalisieren, denn die Zeit dafür ist knapp (Luhmann 1970: 14-15).

4. Ermüdungsphase: Schließlich zeigen sich erste Ermüdungserscheinungen. Es wird sich distanzierter geäußert und Bedenken nehmen größeren Raum ein.

„Bald darauf verliert das Thema seine werbende Kraft. Die Kenner wenden sich von ihm ab. [...] Wer sich jetzt noch mit Verve für das Thema einsetzt, zeigt nur, daß er nicht auf dem laufenden ist. Als Impuls für Veränderungen ist das Thema tot, jedenfalls schwieriger zu beleben als ungeborene Themen, weil seine Geschichte eine Erneuerung blockiert. " (Luhmann 1970: 15)

Für die Ermüdung der Aufmerksamkeit gegenüber dem Thema ist nicht entscheidend, inwieweit das dem Thema zugrunde liegende Problem gelöst wurde (Rössler 2015: 463-465).

Downs macht in seinem viel beachteten Artikel „Up and Down With Ecology“ (1972) ähnliche Beobachtungen wie Luhmann und kommt zu einer vergleichbaren Phaseneinteilung. Durch seinen Begriff ,issue-attention cycle“ weist er bereits darauf hin, dass Thematisierungsprozesse zyklisch zu verstehen sind, dass also der Abschwung des einen mit dem Aufschwung eines anderen Themas korrespondiert, da die Medienöffentlichkeit im Sinne eines Nullsummenspiels eine mehr oder weniger gleichbleibende Zahl von Themen prozessieren kann und muss. Die Betonung der Aufmerksamkeit repräsentiert deren Funktion als die zentrale Währung, in der sich der Stellenwert eines öffentlichen Themas ausdrückt (Rössler 2015: 464). Stärker als Luhmann fokussiert Downs auf das Verhältnis zwischen öffentlicher Aufmerksamkeit und Streitfrage und nimmt nur am Rande Strukturen und Akteur*innen in den Blick. Angesichts dieser Perspektive ist es nicht verwunderlich, dass Downs für den Durchbruch eines Themas nicht das Handeln mutiger 
Pionier*innen als ursächlich herausstellt, sondern eine Aneinanderreihung dramatischer Ereignisse. Im Gegensatz zu Luhmann beschreibt Downs außerdem eine fünfte Phase, die „post-problem stage“ (Downs 1972: 40-41). In dieser letzten Phase ist das Thema nicht mehr, oder wenn dann nur noch punktuell, Teil der öffentlichen Agenda. Allerdings hat die öffentliche Aufmerksamkeit nun eine andere Beziehung zum Thema als vor dessen erstmaligem Erscheinen auf der Agenda. Hat es den issue-attention cycle bereits durchlaufen, kann es jederzeit wieder öffentliche Aufmerksamkeit erregen. Zur Lösung der Streitfrage wurden außerdem, etwa durch ein neues Gesetz oder ein neues Gremium, möglicherweise Strukturen dauerhaft transformiert, die über den Lebenszyklus des issues hinaus Wirkung entfalten. Auch hebt Downs hervor, dass einzelne Aspekte der Streitfrage mit einem neuen issue verknüpft werden können, das gerade erst auf die Agenda gelangt (ist) (Downs 1972: 39-41).

Aufbauend auf Luhmann und Downs wurden zahlreiche weitere Themenzyklusmodelle entwickelt. Sie beruhen auf ähnlichen Überlegungen, reichern diese aber durch Einzelfallstudien mit empirischen Befunden an und/oder verknüpfen sie mit Ansätzen anderer Disziplinen. Das grundlegende Muster eines Themenzyklus - je nach empirischem Untersuchungsgegenstand und theoretischer Einbettung in unterschiedlichen Ausprägungen - wird dabei immer wieder bestätigt (Rössler 2015: 464): Die Aufmerksamkeit eines Teilsystems (Politik, Medien, Öffentlichkeit) gegenüber einem Thema schwankt zunächst auf niedrigem Niveau. Es folgt der Durchbruch und mit ihm ein steiler Anstieg der Aufmerksamkeit. Nach einer meist schmalen Spitze fällt die Aufmerksamkeit im weiteren Verlauf - teils stufenweise, teils in Wellen - wieder ab (Miltner und Waldherr 2013: 273). Modifiziert werden die ursprünglichen Modelle primär in der Abgrenzung, Charakterisierung und Dauer der einzelnen Phasen (vgl. bspw. Rössler 2015: 463-465; Kolb 2005: 41-100; Mathes und Pfetsch 1991; Pfetsch 1986; Wien und Elmelund-Præstekær 2009; Berens 2001; McComas und Shanahan 1999). Unterschiede treten insbesondere bezüglich der letzten Phase auf, bei der einige Studien ein gegenüber der Latenzphase erhöhtes Niveau der Aufmerksamkeit feststellen, während in anderen Studien die Aufmerksamkeit auf das Niveau der Latenzphase zurückfällt. Auf der Grundlage einer umfassenden interdisziplinären Synopse von Lebens- und Themenzyklusmodellen gelangt Kolb (2005: 301-302) für sein daraus abgeleitetes, eigenes Modell zu der vergleichsweise wertungsfreien Phasendenomination mit Latenz-, Aufschwung-, Etablierungs-, Abschwung- und Marginalisierungsphase (siehe Abb. 3.1). 


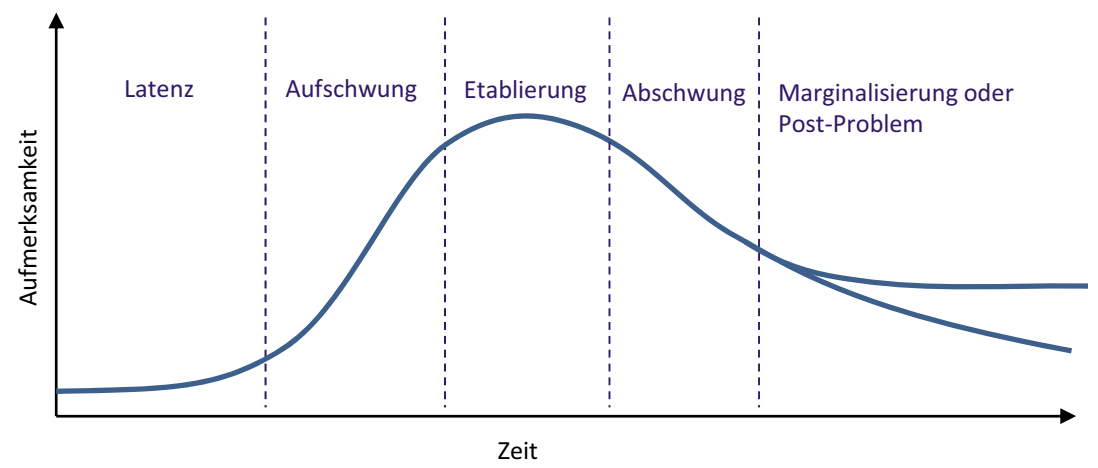

Abb.3.1 Klassische Aufmerksamkeitskurve in Themenzyklusmodellen (Eigene Darstellung basierend auf Luhmann (1970), Downs (1972) und Kolb (2005: 301-302))

Newig (2004) führt aus, dass Themenzyklen ab einer gewissen Kategorienbreite bzw. Großmaßstäblichkeit des Themas nicht mehr auszumachen sind. Stattdessen zeigen solche breiten Politikfelder, wie bspw. „Umwelt“ oder „Bildung “, lediglich langsame Veränderungen in unterschiedlich verlaufenden Aufmerksamkeitskurven. Allerdings ist es möglich, dass eine Vielzahl einzelner Sub-Themen (bspw. Waldsterben, Ozonloch, Treibhauseffekt) jeweils Themenzyklen erleben, die die Aufmerksamkeit gegenüber dem übergeordneten Thema (bspw. Ökologie) immer wieder „aufladen“ (Newig 2004: 155-156) (siehe Abb. 3.2). Damit lässt sich erklären, warum die in Downs' Werk ,Up and Down With Ecology“ (1972) formulierte These, die Aufmerksamkeit der US-amerikanischen Öffentlichkeit gegenüber dem Thema Ökologie würde bald abflauen, sich als offensichtlich falsch erwiesen hat. Nämlich nicht aufgrund der generellen Untauglichkeit des Modells, sondern weil das Thema Ökologie für das Modell zu breit angelegt war.

Häufig werden die Themenzyklusmodelle erweitert, indem sie mit anderen Theorien oder Modellen verknüpft werden. Grundsätzlich ist dabei festzustellen, dass zum einen die (unterschiedlich gehandhabte) systematische Phaseneinteilung des Modells, zum anderen aber auch die fehlende Weiterentwicklung in Richtung eines umfassenden und wirklich erklärenden theoretischen Programms eine gute Grundlage für die Integration von Theorien bietet, deren Stärken anders gelagert sind. Als Beispiele seien Verknüpfungen mit Agenda Setting- oder Framing-Modellen aus den Medien- und Kommunikationswissenschaften (bspw. Brosius und Eps 1993), Agenda Setting-Modellen aus der Politikwissenschaft 


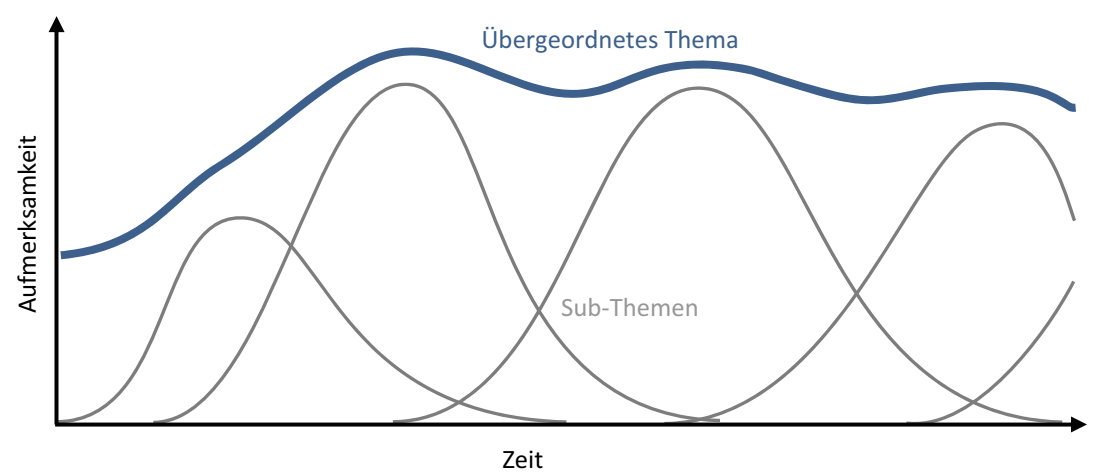

Abb.3.2 Verlauf der Aufmerksamkeit eines übergeordneten Themas (blau) (Eigene Darstellung basierend auf Newig (2004: 156))

(bspw. Paasch-Colberg 2017), Lebenszyklusmodellen aus der Wirtschaftswissenschaft (bspw. Kolb 2005) oder auch mit issue-Management Ansätzen aus der Unternehmenskommunikation (bspw. Lütgens 2001; Ingenhoff 2004) genannt. Insbesondere letztere Theorieverknüpfungen deuten ein weiteres Anwendungsgebiet des Modells an, denn es dient oft als Grundlage für die Entwicklung eines Interventionsinventars, mit dem der Thematisierungsprozess beeinflusst werden kann (Rössler 2015: 463-465).

\section{Schlussfolgerungen}

Das Themenzyklusmodell - mit unterschiedlich segmentierten Phasen von Latenz, Aufschwung, Etablierung und Abschwung - stellt bis heute eine beliebte und vielfach bewährte Heuristik dar, mit deren Hilfe empirisches Material strukturiert und in jeweils vergleichbare Segmente aufgeteilt werden kann. Erkenntnisse zu einzelnen Aspekten der Themenkarriere können so zielgerichtet gewonnen und verallgemeinert werden. Häufig kommt dabei eine quantitative Analyse der Aufmerksamkeit gegenüber einem Thema im Zeitverlauf - bspw. auf Basis von Zeitungsartikeln zur Prüfung der Themenzyklushypothese zum Einsatz. Beides - eine quantitative Analyse auf der Basis von Publikationsdaten sowie eine Segmentierung der Themenkarriere zu Analysezwecken - erfolgt auch im Rahmen dieser Arbeit (siehe Kp. 6-8), um evaluieren zu können, ob und in welcher Form Themenkarrieren auch in der Planungswissenschaft vorliegen. Darüber hinaus sind die dargelegten Erkenntnisse zu den Mechanismen, die im Zuge der Phasen bzw. der Phasenübergänge ablaufen, für die Beantwortung der hier verfolgten Forschungsfrage 
aufschlussreich. Dabei ist insbesondere die strukturierende Wirkung einer Themenkarriere für das Handeln verschiedener Akteur*innengruppen im Sinne Luhmanns zu nennen. So ergeben sich aus den jeweiligen strategischen Interessen dieser Gruppen in Abhängigkeit von dem Stadium der Themenkarriere unterschiedliche Handlungsoptionen, die wiederum für die Entwicklung des Themas maßgeblich sind. Die allen Themenzyklusmodellen zugrundeliegende Hypothese, dass die Kommunikation über das Thema ein Eigenleben im öffentlichen Diskurs entfaltet, das sich abgekoppelt von einer - wie auch immer $\mathrm{zu}$ bestimmenden - ,objektiven “ Relevanz entwickelt, ist Ausgangspunkt dieser Arbeit.

\subsection{Agenda Setting in den Medien- und Kommunikationswissenschaften}

Die Medien- und Kommunikationswissenschaften befassen sich primär mit der Rolle des Mediensystems im öffentlichen und politischen Diskurs. Demnach vermittelt das Mediensystem zwischen den Teilsystemen der Gesellschaft (bspw. Wirtschaft, Wissenschaft) und der Politik und übernimmt eine entscheidende Rolle bei der Selektion und Definition der relevanten, lösungsbedürftigen Probleme sowie bei der Herstellung gesellschaftlicher und politischer Aufmerksamkeit für diese Probleme (Paasch-Colberg 2017: 52). Während Themenzyklusmodelle den Verlauf der öffentlichen Aufmerksamkeit gegenüber einem Thema beschreiben und dabei grundlegende Mechanismen dieses Verlaufs auf der Makroebene identifizieren, gehen die hier geschilderten Agenda Setting-Ansätze ins Detail und analysieren die Themenproduktion durch mediale Akteur*innen. Die Stärke der medien- und kommunikationswissenschaftlichen Agenda SettingAnsätze liegt daher in der Identifizierung und Erklärung von Mechanismen der Themenselektion und -konstruktion. Analog zu den Themenzyklusmodellen wird Aufmerksamkeit - der Medien, Politik oder Gesellschaft - als eine begrenzte Ressource verstanden, um welche die Themen miteinander in Konkurrenz stehen, denn, so konstatiert Zhu (1992: 825): „, The addition of any new issue onto the public agenda is at the cost of other issues “. Agenda lässt sich hier verstehen als ,a list of issues and events that are viewed at a point in time as ranked in a hierarchy of importance " (Rogers und Dearing 1988: 565).

Ausgangspunkt für das Agenda Setting-Konzept ist Bernard C. Cohens Hypothese: „[the press] may not be successful much of the time in telling people what to think, but it is stunningly successful in telling its readers what to think about" (Cohen 2015 [1963]: 13). McCombs und Shaw (1972: 177) wiesen schließlich 
nach, dass die Themengewichtung in der medialen Berichterstattung einen Einfluss darauf hat, welche Themen die Rezipient*innen für wichtig erachten und führten den Begriff des Agenda Setting ein (McCombs und Shaw 1972; PaaschColberg 2017: 34). Diese in zahlreichen Studien bewiesene These (für einen Überblick siehe Wolfe et al. 2013: 178) markierte den Beginn eines Paradigmenwechsels weg von dem Fokus auf das direkte Übertragen von Meinungen und Einstellungen bezüglich eines Themas durch die Medien auf die Rezipient*innen - welches nur in wenigen Fällen beobachtet werden konnte - und hin zur Übertragung einer Agenda bzw. einer Auswahl von Themen, die für wichtig erachtet werden (Bulkow und Schweiger 2013: 172; Wolfe et al. 2013: 178; Zahariadis 2016: 4).

Zentral für das Agenda Setting ist demnach die Konstruktion von Wichtigkeit oder Salienz eines Themas und weniger dessen Kontextualisierung und Bewertung. Nichtsdestotrotz nimmt Agenda Setting Einfluss auf persönliche Einstellungen und Bewertungsmaßstäbe. Indem sich die Salienz eines Themas bei den Rezipient*innen erhöht, wird es bei späteren Bewertungen oder Urteilsbildungen stärker ins Gewicht fallen. Lang und Lang (1981: 449) kommen daher zu dem Schluss, dass sich die Herstellung von Salienz und die Übertragung von Meinungen nicht trennen lassen:

„What people think may not be as easily separable from what they think about as the various formulations of agenda-setting have implied. On the contrary, many differences in opinion originate from the different weights people attach to elements in a complex situation."

Durch die Herstellung von Salienz bestimmter Themen und Themenattribute beeinflussen die Medien also die Gewichtung gesellschaftlicher Probleme in der öffentlichen Kommunikation. Über diesen Umweg werden Bewertungsmaßstäbe verschoben und dadurch soziales Handeln und politische Entscheidungsprozesse strukturiert (Paasch-Colberg 2017: 36-38).

Verschiedene Forschungstraditionen im Rahmen der Medien- und Kommunikationswissenschaften beschäftigen sich mit den Prozessen und Faktoren, die maßgeblich für die mediale Selektion eines Themas sind - darunter insbesondere Forschungen zu Nachrichtenwert, Gatekeeper*innen und issue obstrusiveness (vgl. Eichhorn 2005; Rauchenzauner 2008; Vonbun et al. 2016). Die häufig identifizierten Faktoren lassen sich unterscheiden in solche, die sich auf das Thema beziehen und solche die sich auf das Medium bzw. die Journalist*innen beziehen. Anhand der Charakteristika des Mediums oder der Journalist*innen lässt sich abschätzen, ob und in welcher Form ein bestimmtes Thema oder Ereignis 
aufgegriffen wird und welchen Einfluss die Berichterstattung haben wird. Zu diesen Eigenschaften zählen bspw. der Produktionszyklus und die Geschwindigkeit der Berichterstattung (bspw. Wochenmagazin oder Online-Nachrichtenportal), der thematische Zuschnitt (bspw. fachspezifisch oder allgemein) sowie die Funktion innerhalb der Medienlandschaft (bspw. nationale Meinungsführung oder Lokalberichterstattung) (vgl. Vonbun et al. 2016: 1056-1059).

Die auf das Thema bezogenen Faktoren der medialen Selektion lassen sich differenzieren in (1.) Nachrichtenfaktoren, die einem Thema zugeordnet werden und die im Rahmen der Nachrichtenwerttheorie häufig als „objektspezifisch“ bezeichnet werden, sowie in (2.) Nachrichtenwert, der durch die Gewichtung der Nachrichtenfaktoren auf Seiten der Rezipient*innen entsteht, der also je nach Rezipient*in variiert (Schulz 1990: 30). Viele Arbeiten beschäftigen sich mit der Identifizierung und Auflistung von Nachrichtenfaktoren (für eine Übersicht, siehe Harcup und O'Neill 2017; Schultz 2007; Rauchenzauner 2008; Maier et al. 2018). Exemplarisch seien hier die Faktoren Neuigkeitswert/Zeitlichkeit, Überraschungseffekt, Nähe/Identifikation, Konflikthaftigkeit, Bekanntheit/Prominenz und Illustrierbarkeit genannt. Je stärker Journalist*innen diese Faktoren erfüllt sehen, so die Theorie, umso wahrscheinlicher ist die Berichterstattung und umso größer ist voraussichtlich der Raum, der dieser Berichterstattung eingeräumt wird.

Aus der Agenda Setting-Perspektive heraus wurde das sogenannte ,secondlevel agenda setting“ - auch „,attribute agenda setting “ oder ,framing“ - entwickelt, das unterhalb der Themenebene ansetzt und die Attributionen eines Themas untersucht (Paasch-Colberg 2017: 37; Kiousis et al. 1999: 414-416). So befasst sich der Framing-Ansatz gemäß Rössler (2015: 473) „mit der Art und Weise, in der Sachverhalte in der öffentlichen Diskussion, gerahmt ' werden, $d$. h., welche Aspekte betont und welche weggelassen, welche Bezüge hergestellt und welche Parallelen gezogen werden ". Typischerweise wird das Framing-Konzept auf die Rahmung bestimmter Fakten oder Ereignisse bezogen, genauso können aber auch Themen mit bestimmten Attributen und anderen Themen verknüpft und somit gerahmt werden (Potthoff 2012: 20). Nach einer verbreiteten Definition von Entman (1993: 52) bedeutet Framing,

„[...] to select some aspects of a perceived reality and make them more salient in a communicating text, in such a way as to promote a particular problem definition, causal interpretation, moral evaluation, and/or treatment recommendation for the item described. “ 
Während also der Agenda Setting-Ansatz die Selektion von Themen und das Herstellen von deren Salienz in der Öffentlichkeit fokussiert, zielt der FramingAnsatz auf die Perspektivierung der ausgewählten Themen (Kiousis et al. 1999: 414-416). Durch die Hervorhebung, Auslassung und Selektion bestimmter Attribute wird ein bestimmtes Deutungsmuster etabliert, das wiederum die Aufmerksamkeit gegenüber dem Ereignis sowie dessen Bewertung beeinflusst. Diese Verknüpfung zwischen dem Framing eines Ereignisses und der Herstellung von Aufmerksamkeit bzw. dem Agenda Setting wird im Rahmen der Forschung $\mathrm{zu}$ „Compelling Arguments“ betont, die zeigt, dass die zunehmende Salienz bestimmter Themenattribute auch die Salienz des Themas selbst erhöht (Wolfe et al. 2013: 178; McCombs und Ghanem 2001).

Eine Grundidee des Agenda Setting- sowie des Framing-Konzeptes ist, dass Agendas und Frames nicht von selbst emergieren, sondern von gesellschaftlichen Akteur*innen (strategisch) formuliert und eingesetzt werden. In manchen Diskursen werden bspw. Themenunternehmer*innen ausgemacht, also professionelle Akteur*innen und Organisationen, die bestimmte Deutungsmuster forcieren und bestimmte Deutungsprozesse in Gang setzen wollen (Waldherr 2008a: 174). Hier setzen auch Themenmanagement- und PR-Ansätze an, die auf die Strategien fokussieren, die zum Einsatz kommen, um diejenigen Themen in der Berichterstattung zu verankern, die dem eigenen Programm nützen oder dem politischen Gegner schaden. Komplementär zu diesem Verständnis von Agenda Setting verhält sich das „Agenda Cutting“, also der Versuch diejenigen Themen aus der Berichterstattung herauszuhalten oder zu entfernen, die für das eigene Programm nachteilig bzw. für das gegnerische Programm vorteilhaft sind. „Agenda Surfing " bezeichnet schließlich das Aufgreifen eines aufkommenden Themas in der öffentlichen Kommunikation, um Aufmerksamkeit für das eigene Programm zu erzeugen und sich politisch zu profilieren (Brettschneider 2014: 653).

Verschiedene Arbeiten verknüpfen Ansätze aus der Agenda SettingForschungstradition mit Themenzyklusmodellen. Von der Beobachtung ausgehend, dass sich Durchbruch und Aufschwung eines Themas häufig auf ein Schlüsselereignis zurückführen lassen, analysieren Brosius und Eps (1993) die durch ein Ereignis induzierten Agenda Setting-Mechanismen. Sie weisen inhaltsanalytisch nach, wie sich im Nachgang eines Schlüsselereignisses die journalistischen Selektionskriterien verändert haben (Scheufele 2016: 289). So wird durch ein Schlüsselereignis entweder ein neues Thema geschaffen oder einem schon bekannten Thema eine neue Dimension verliehen. In beiden Fällen müssen Journalist*innen und Rezipient*innen neue Maßstäbe für die Bewertung und Einordnung des Ereignisses entwickeln. Dieses Bedürfnis nach Orientierung zieht zum einen eine stärkere Achtsamkeit gegenüber vergleichbaren Ereignissen nach 
sich und führt zum anderen zu einer intensivierten Suche nach Informationen über das Ereignis und verwandte Themen durch die Journalist*innen (Rauchenzauner 2008: 21-24). In der Folge wird die einschlägige Berichterstattung stark ausgeweitet, was den Eindruck einer außergewöhnlichen Häufung entsprechender Ereignisse und einer besonderen Wichtigkeit des Themas vermittelt, auch wenn sich anhand statistischer Daten nachweisen lässt, dass sich an der tatsächlichen Situation nichts geändert hat (ebd.: 23). Hinzu kommt, dass ein Schlüsselereignis und die diesbezügliche Berichterstattung gesellschaftliche Auswirkungen haben, die wiederum zu einer Etablierung des Themas führen, etwa wenn die Politik mit Entscheidungen auf die öffentliche Erregung reagiert oder wenn Interessengruppen die Gelegenheit ergreifen, das Thema voranzutreiben (ebd.: 25).

Mathes und Pfetsch (1991) arbeiten die Rolle der Massenmedien in den unterschiedlichen Phasen heraus und zeigen, dass die Berichterstattung über ein Thema teilweise von Spezial- und Alternativmedien ausgeht. Diese rücken das Thema als erstes in den Fokus und bereiten es für den Diskurs in einer Teilöffentlichkeit auf, von wo es - vermittelt durch ein Transmitter-Medium, das zwischen Alternativ- und Mainstream-Medien verortet ist - schließlich durch die Mainstream-Medien aufgegriffen wird (ebd.: 53). Dieser medieninterne „spillover-effect“ (ebd.: 42-57) wird konzeptionell auch unter ,intermedia agenda setting “ gefasst (Rössler 2015: 464). Der Ansatz von Mathes und Pfetsch betont die Rolle der Mainstream- oder Leitmedien insbesondere in der Durchbruchphase. Indem andere Journalist*innen diese als Informationsquelle und Bezugsrahmen für ihre eigene Berichterstattung nutzen, diffundieren deren Themengewichtungen und Frames. Aufgrund dieser Ausstrahlungswirkung in das Mediensystem haben sie eine besondere Bedeutung für die Durchsetzung von Themen, weshalb sie primäres Ziel für Interessengruppen sind, die ein Thema auf die Agenda heben wollen (Rössler 2015: 464).

Ihren Erkenntnissen folgend modifizieren Mathes und Pfetsch (1991) Luhmanns Themenkarriere in mehreren Punkten. In der Latenzphase existiert das Problem bereits, aber dessen Wichtigkeit wurde noch nicht erkannt. Sobald die Alternativmedien das Thema aufgreifen endet die Latenzphase und die Vorbereitungsphase beginnt. Nun widmen die Alternativmedien dem Problem mehr und mehr Aufmerksamkeit und bereiten so den Spillover vor. In der Durchbruchoder Spillover-Phase übernehmen die etablierten Medien das Thema, was eine Aufschwungphase initiiert, in der mehr und mehr Medien das Thema immer ausführlicher behandeln, bis schließlich die Klimaxphase erreicht ist, in der das Thema die Medien- und Politik-Agenda beherrscht. Die politische Entscheidung zur (vermeintlichen) Lösung des Problems läutet die Abschwungphase ein. Die 
Medien verlieren das Interesse, selbst wenn die politische Entscheidung das Problem nicht gelöst, sondern nur aufgeschoben hat (Mathes und Pfetsch 1991: 57).

Wolfsfeld (2000) problematisiert den Umstand, dass die Massenmedien und die Öffentlichkeit nach einer Phase der Euphorie unabhängig von der Lösung des Problems das Interesse verlieren und sich anderen Problemen zuwenden. Sie seien ab einer gewissen Dauer an einem Abschluss des Problems interessiert (,,looking for closure"), was angesichts langer und komplizierter Politikprozesse - sie untersucht „political waves“ im Friedensprozess in Israel - zu einem verkürzten und unrealistischen Verständnis der wirklichen Streitfragen führe (Wolfsfeld 2000: 248). Wolfsfeld und Sheafer (2006) fokussieren auf die Akteur*innen und verfolgen deren Rolle und Handlungsspielräume in den unterschiedlichen Phasen des Themenzyklus. Dabei heben sie insbesondere die Aktivierung einer Vielzahl von Akteur*innen zu Beginn des Themenzyklus als demokratiefördernden Effekt hervor. In der Phase erhöhter Aufmerksamkeit konkurrieren politische Akteur*innen um Berichterstattung, wobei unter anderem der Grad, mit dem die Akteur*innen mit dem Thema in Verbindung gebracht werden können, sowie ihr politischer Rang (,political standing“) entscheidende Faktoren für ihre mediale Präsenz darstellen (Wolfsfeld und Sheafer 2006: 333).

Eine Reihe von Ansätzen hebt die besondere Bedeutung des Framings als zentralen Mechanismus innerhalb von Themenzyklen hervor (vgl. Nisbet und Huge 2006; Gottlieb 2015; Miller und Riechert 2001). Gemäß Miller und Riechert (2001: 111) setzt mit dem Aufschwung eines Themas in der Definitions/Konfliktphase der Wettbewerb um dessen Rahmung ein, indem sich die konkurrierenden Stakeholder*innengruppen darum bemühen, ihr Deutungsmuster möglichst prominent zu platzieren. Dieser Kampf um die Deutungshoheit hat für sich Nachrichtenwert und kann eine der Hauptantriebskräfte der wachsenden Aufmerksamkeit werden (Miller und Riechert 2001: 111). In der Resonanzphase resoniert ein Frame besonders gut mit den Werten und Erlebnissen der Öffentlichkeit und es setzt ein zyklischer Prozess ein, bei dem die resonierenden Attributionen diffundieren. Gruppen, die ihre Position durch den resonanten Frame gestärkt sehen, nutzen dessen Attributionen, um für ihre Sichtweise zu werben. Aber auch Gruppen, die ihre Position geschwächt sehen, müssen sich der Terminologie des resonanten Frames anpassen, um überhaupt wahrnehmbar dagegenhalten zu können (Miller und Riechert 2001: 111-112). In der Gleichgewichts- oder Resolutionsphase dominiert ein Frame und bestimmt die Entscheidungen der Politik: 
„The winning frame can so dominate that others are delegitimized and given no credence in the media and public discourse. When this occurs the dominant frame could be said to be acting hegemonically, rendering, natural' the prevailing definition of the situation. “ (Miller und Riechert 2001: 112)

Opponierende Stakeholdergruppen sind nun zwar weitestgehend verstummt, neue Ereignisse können ihnen aber wieder Gelegenheit verschaffen, ihre Sichtweise zu präsentieren, sodass das entstandene Gleichgewicht erneut in Frage gestellt wird (Miller und Riechert 2001: 112). An diesem Punkt - dem Kampf unterschiedlicher Gruppierungen um die Deutungshoheit und die öffentliche Meinung - setzen die politikwissenschaftlichen Theorien und Modelle an (siehe Kp. 3.4).

\section{Schlussfolgerungen}

Die medien- und kommunikationswissenschaftlichen Agenda Setting-Ansätze halten einige Erkenntnisse bereit, die eine Berücksichtigung im empirischen Teil dieser Arbeit erfordern. Zunächst ist die Übertragbarkeit von Mechanismen der Thematisierung zu prüfen. So könnten etwa die wechselseitigen Spillover-Effekte zwischen Spezial- und Mainstream-Diskursen, aber auch die Veränderung der Selektionskriterien sowie des betriebenen Rechercheaufwands im Zuge einer Themenkarriere möglicherweise auf die Wissenschaft übertragen werden. Ebenso ist der Zusammenhang zwischen Framing und Salienz hervorzuheben: Einerseits wird durch die Etablierung eines Frames die Aufmerksamkeit gegenüber dem Thema (Salienz) beeinflusst, andererseits setzt sich die Salienz eines Themas aus der Aufmerksamkeit gegenüber den verschiedenen Themenattributen im Rahmen des vorherrschenden Deutungsmusters (Frames) zusammen. Insbesondere in Betracht zu ziehen ist die Erkenntnis, dass normative Bewertungen im öffentlichen Diskurs zwar nicht unbedingt direkt übertragen werden können, dass aber sehr wohl durch die Salienz und das Framing von Themen ihre Gewichtung bei den Rezipient*innen beeinflusst wird, sodass indirekt Bewertungsmaßstäbe verschoben werden. Dabei ist zu beachten, dass die Medien hier in der Regel als Vermittler*innen auftreten, die ein externes Publikum beeinflussen. Die Übertragung der Konzepte auf die Wissenschaft erfordert daher gewisse Anpassungen, da Wissenschaftler*innen im Normalfall ihr eigenes Publikum sind. Allerdings operiert gerade die Planungswissenschaft nicht isoliert von der Gesellschaft, sondern übernimmt und überträgt - vermittelt über die Ausbildung, Gutachten, angewandte Forschung etc. - Salienzen, Frames und dementsprechend Bewertungsmaßstäbe. Eine Nutzbarmachung der in diesem Kapitel dargelegten Erkenntnisse für die Planungswissenschaft und die Raumplanung - auch über diese Arbeit hinaus - erscheint daher als fruchtbar. 


\subsection{Agenda Setting in der Politikwissenschaft}

Nicht nur die medien- und kommunikationswissenschaftliche, sondern auch die politikwissenschaftliche Agenda Setting-Forschung beschäftigt sich mit der Herstellung von Aufmerksamkeit gegenüber Streitfragen. Trotz offensichtlicher Überschneidungen haben beide Traditionen jeweils eigene Literaturkorpora und spezifische Fragestellungen entwickelt. Während die Medien- und Kommunikationswissenschaften die Rolle der Medien in den Vordergrund rücken, sind diese im Rahmen der Politikwissenschaft lediglich eine von mehreren Institutionen des politischen Diskurses und werden mitunter auf die Funktion eines Indikators oder Vermittlers bestimmter Positionen reduziert (Wolfe et al. 2013: 180; Scheufele 2003: 48-89). Charakteristisch für die Politikwissenschaft ist es, Agenda Setting als einen zentralen Bestandteil des Politikprozesses zu verstehen, der also vor dem Hintergrund des Zustandekommens einer politischen Entscheidung zu analysieren ist. Für die Fragestellung dieser Arbeit sind insofern Aufschlüsse zu erwarten, als dass das - auch in der Wissenschaft angelegte - Ringen von Akteur*innen um Deutungshoheit und Aufmerksamkeit im Diskurs in den Mittelpunkt der Betrachtung gestellt wird.

Weil im Gegensatz zum medienwissenschaftlichen Verständnis seltener das einseitige, aktive Setzen der öffentlichen und politischen Agenda (durch die Medien) fokussiert wird und stattdessen ganz grundsätzlich nach der Vielzahl von Faktoren und Akteur*innen gefragt wird, die zur Entstehung der Politikagenda beitragen, wird diese Forschungstradition teilweise auch unter „Agenda Building “ oder „Agenda Formation“ geführt (Rogers und Dearing 1988: 556; Wolfe et al. 2013: 175). Zwar betrachtet auch die Politikwissenschaft das Konkurrieren um die knappe Ressource Aufmerksamkeit - bei Wolfe et al. (2013: 179) "the politics of attention“. Allerdings fokussiert sie dabei auf die Entstehung von politischen Entscheidungen, wobei insbesondere die Aufmerksamkeit derjenigen (politischen) Akteur*innen in den Fokus rückt, die ebenjene Entscheidungen treffen. Damit einher geht die stärkere Betonung der Rolle von Eliten im Agenda Setting- und Politikprozess (ebd.: 177).

Birkland (2007: 77) umreißt die Gegenstände und Ziele dieser Forschungstradition folgendermaßen:

„The study of agenda setting is a particularly fruitful way to begin to understand how groups, power, and the agenda interact to set the boundaries of political policy debate. Agenda setting, like all other stages of the policy process, does not occur in a vacuum. The likelihood that an issue will rise on the agenda is a function of the issue itself, the actors that get involved, institutional relationships, and, often, random social and political factors that can be explained but cannot be replicated or predicted. But 
theories of agenda setting, coupled with better and more readily available data, are enabling researchers to understand why and under what circumstances policy change is likely to occur."

Gemäß Zahariadis (2016: 5) lässt sich Agenda im politikwissenschaftlichen Verständnis als eine „contextual list of actionable government priorities“ fassen, wobei hier drei Elemente zum Tragen kommen sollen: contextual, actionable und priorities. Die Geltung und Entstehung einer Agenda ist demnach nur vor dem Hintergrund ihres zeitlichen und gesellschaftlichen Kontextes zu verstehen. Sie beschränkt sich auf solche Themen und Entscheidungen, die die Akteur*innen glauben beeinflussen zu können. Damit also ein Thema überhaupt auf die Agenda gelangen kann, bedarf es einer Rahmung als politisch lösbares Problem (Weingart et al. 2000: 263). Außerdem gibt es eine Priorisierung, denn sowohl die Öffentlichkeit als auch politische Akteur*innen verfügen über knappe Ressourcen und müssen Selektionsentscheidungen treffen (Paasch-Colberg 2017: 52; Zahariadis 2016: 5-6).

Es kann zwischen Agenden verschiedener Regierungsebenen, verschiedener Arenen sowie verschiedener Akteur*innen und Akteur*innenkreise differenziert werden. Häufig wird eine Unterscheidung dreier Agenda-Ebenen vorgenommen: Die systemische Agenda enthält alle Ideen, die politische Akteur*innen grundsätzlich in dem untersuchten Kontext für entscheidungsrelevant erachten. Interessengruppen versuchen ihr Thema von dort auf die institutionelle Agenda zu heben, auf der sich nur diejenigen Themen befinden, die von den maßgeblichen Entscheidungsträger*innen aktiv und sorgfältig betrachtet und beurteilt werden müssen (Cobb und Elder 1972: 85-86). Die Entscheidungsagenda enthält schließlich nur jene Themen, über die eine konkrete Entscheidung ansteht (Birkland 2007: 63-65).

Agenda Setting lässt sich allgemein verstehen als ,the process by which problems and alternative solutions gain or lose public and elite attention" (Birkland 2007: 63-65). In der Regel wird aber das Aufsteigen auf die institutionelle Agenda betrachtet, also gemäß der bekannten Definition von Cobb und Ross (1997: 3) "the politics of selecting issues for active consideration ". Von diesem Grundverständnis ausgehend scheiden sich Agenda Setting-Ansätze an der Frage, ob analytisch zwischen Problemdefinition und Agenda Setting differenziert wird. In dem expansiven Verständnis geht es nämlich nicht nur darum, $o b$ ein Thema Aufmerksamkeit erhält, sondern auch um die Fragen, welches Deutungsmuster und welche möglichen Lösungen damit verknüpft werden (Birkland 2007: 63; Dery 2000: 37; Cobb und Ross 1997: 3-4; Schattschneider 1960, 1975). Diesem Verständnis ist auch Zahariadis (2016: 6) zuzurechnen, der Agenda Setting 
anknüpfend an sein oben dargestelltes Agenda-Verständnis als ,the process of turning public interests into actionable government priorities " definiert. Weiterhin bringt Birkland (2007: 63) die Ableitung von Handlungsoptionen in einen Zusammenhang mit der Selektion und dem Framing des Problems:

\begin{abstract}
„Even when an issue gains attention, groups must fight to ensure that their depiction of the issue remains in the forefront and that their preferred approaches to the problem are those that are most actively considered. [...] [T] he group that successfully describes a problem will also be the one that defines the solutions to it, thereby prevailing in policy debate. " (Birkland 2007: 63)
\end{abstract}

Im Rahmen der auf Lasswell (1956) zurückgehenden Politikzyklusmodelle stellt Agenda Setting eine Stufe oder Phase im Rahmen eines idealtypischen Politikprozesses dar. Die heute bekannteste Version des Politikzyklus (,policy cycle“) wurde maßgeblich von Jones (1970) und Anderson (1975) entwickelt und umfasst die Phasen „Problem Definition“, „Agenda Setting“, „Policy Formation“ (oder „Formulation“), „Adoption“, „Implementation“ und „Evaluation“ (Jann und Wegrich 2014: 101) (siehe Abb. 3.3).

Die kreisförmige Betrachtung verdeutlicht, dass Politikprozesse in der Regel keine eindeutigen Anfänge und Abschlüsse aufweisen, sodass auch die Phase des Agenda Setting stets vor dem Hintergrund bestehender Politikprozesse betrachtet werden muss (Blum und Schubert 2018: 159). So stellt eine umgesetzte Policy selten die finale Lösung eines Problems dar. Eher ist sie als Bestandteil neuer Herausforderungen und neuer Policies zu sehen. Auf dieses Merkmal haben insbesondere Hogwood und Peters mit ihrem Ansatz der "policy succession" hingewiesen. Demnach stoßen „neue" Policies selten in ein Politikvakuum, sondern es besteht bereits ein „well-occupied or even crowded tablet of existing laws, organizations and clients" (Hogwood und Peters 1982: 225-226). Neue Policies ergänzen oder modifizieren bestehende Policies, können aber auch mit ihnen konkurrieren oder negativ interagieren (Jann und Wegrich 2014: 106). Bereits etablierte Policies haben deshalb einen wesentlichen Einfluss darauf, ob und in welcher Form Themen auf die Agenda gelangen und neue Policies entstehen (Jann und Wegrich 2007: 45).

Der Vorteil des dargestellten linearen policy cycle ist, dass er eine systematische Prozess-Sicht auf politisches Entscheidungshandeln eröffnet und dieses analog zu den Themenzyklusmodellen - in einzelne, jeweils fallübergreifend vergleichbare Segmente unterteilt. Allerdings wird das Modell insbesondere für seine mangelnde empirische Validität kritisiert. Die einzelnen Phasen folgen nur selten chronologisch aufeinander, sondern verlaufen häufig simultan oder kehren 


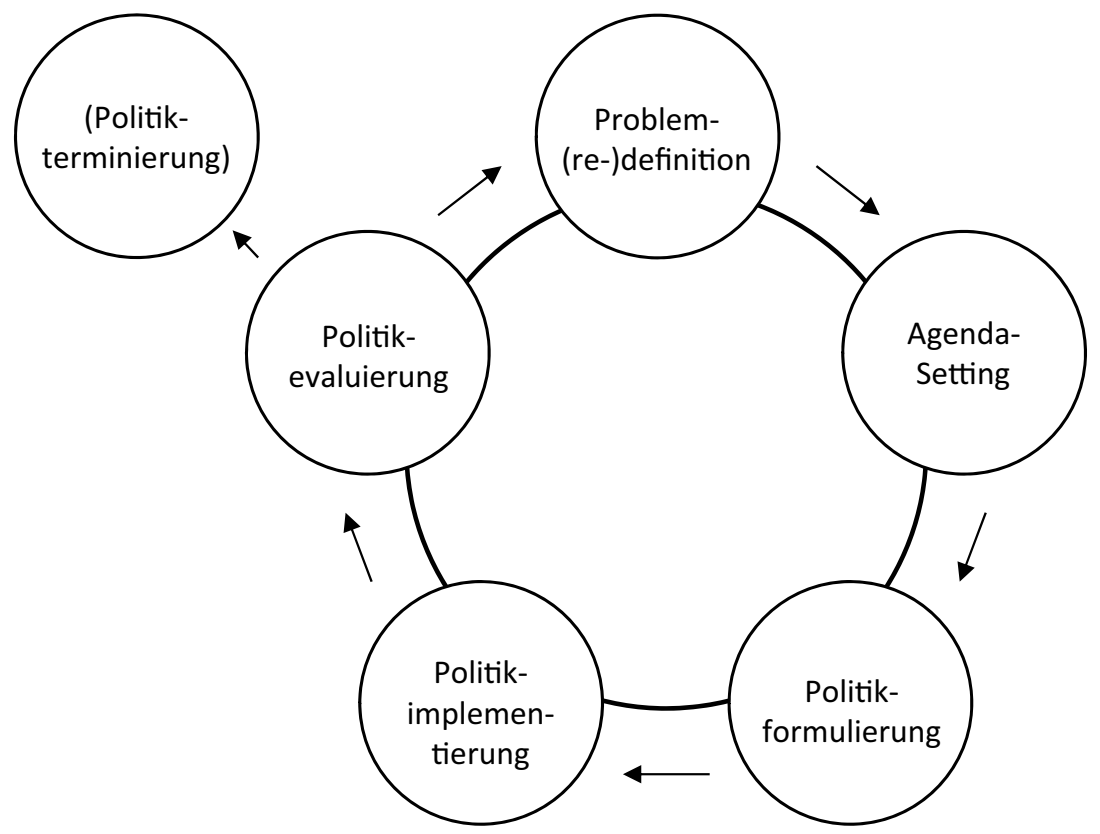

Abb.3.3 Gängige Darstellung des Politikzyklus (Eigene Darstellung nach Jann und Wegrich (2014: 106))

ihre Richtung um (Schmid-Petri 2012: 52-53). Zudem ist die Annahme, es gäbe einen singulären Politikzyklus, gemäß Sabatier (2007: 7) eine starke Simplifizierung des „usual process of multiple, interacting cycles involving numerous policy proposals and statutes at multiple levels of government".

Einigkeit herrscht darin, dass das Politikzyklusmodell und die Kritik daran einige herausragende Forschungen inspirierte, die auf das Agenda Setting fokussieren (Sabatier 2007: 7; Jann und Wegrich 2007: 57). Als einflussreichster Ansatz kann Kingdons (1984) „multiple streams“-Modell betrachtet werden. Es basiert auf dem ,garbage can“-Modell für Entscheidungsprozesse in Organisationen, das Cohen et al. (1972) auf Grundlage von Beobachtungen an Universitäten entwickelten. In Abgrenzung zu den rationalen und linearen Politikzyklusmodellen strukturiert hier das zufällige Aufeinandertreffen von Problemen, Lösungen, Akteur*innen und Entscheidungsgelegenheiten die Entscheidungen. Kingdon führte die Idee von ,policy windows“ ein, die sich öffnen, wenn drei 
voneinander unabhängige Ströme - der ,policy stream “ (Lösungen), der „politics stream " (Entscheidungsprozesse) und der „problem stream “ (Problemwahrnehmung) - sich kreuzen (Jann und Wegrich 2007: 47; Kingdon 1984: 174 ff.). So kann ein Thema bzw. ein Problem erst dann auf die politische Agenda gelangen, wenn auch kompatible Lösungen und Entscheidungsprozesse verfügbar sind (Kingdon 1984: 165 ff.). Umgekehrt warten bereits entwickelte Lösungen auf das Hervortreten öffentlichkeitswirksamer Probleme, mit denen sie verknüpft werden können. Ein Interessenvertreter verdeutlicht dies mit einer Interview-Aussage bei Kingdon (1984: 173):

„The underlying goals exist and continue along. You want to do something, and you ask, ,What will work this year? What's hot this year that I can hang this on? ““

Demnach wird jedes Ereignis, dass in Zusammenhang mit dem Thema steht, zu einer Gelegenheit, eine bestimmte Politik voranzutreiben (Kingdon 1984: 182). Das Öffnen eines policy window heißt jedoch nicht, dass es auch genutzt wird. Stattdessen kann sich die Aufmerksamkeit gegenüber einem Problem rasch zugunsten eines anderen verschieben, ohne dass Lösungen für ersteres verabschiedet werden konnten (Cairney und Zahariadis 2016: 87). Um policy windows erkennen und ausnutzen zu können, müssen die Fürsprecher*innen eines Themas vorbereitet sein. Kingdon (1984: 165) benutzt hierfür das Bild des Surfers, der auf eine Welle wartet: "If you're not ready to paddle when the big wave comes along, you're not going to ride it in ". Hierzu gehört, dass die Ideen und Netzwerkkontakte bereits entwickelt sind, ,bevor die Chance zu ihrer Realisierung tatsächlich eintritt" (Ibert und Gailing 2016: 397). Neben einer guten Vorbereitung bedarf es aber auch eines gewissen Geschicks, um eine Aufmerksamkeitswelle reiten zu können (Kingdon 1984: 181).

Kingdon (1984: 179-183) führt außerdem das Bild des „policy entrepreneur" ein. Damit ein Thema nichtetablierter Gruppen auf die politische Agenda avanciert, bedarf es häufig Persönlichkeiten, die die Thematisierung mehr oder weniger professionell vorantreiben. Solche politischen Unternehmer*innen investieren Ressourcen - Zeit, Energie, Reputation und Geld - in die Thematisierung. Sie versprechen sich von der Durchsetzung des Themas auf der öffentlichen und politischen Agenda zukünftige Gewinne in Form von materiellen oder solidarischen Vorteilen (ebd.: 179). Ein zentraler Bestandteil des entrepreneurship ist der „Aufbau von Netzwerken und Kommunikationsinfrastrukturen, die für das Thema mobilisierbar und organisierbar sind" (Pfetsch 1994: 17). 
Der Politologe Sabatier (1988) fokussiert zur Erklärung von Veränderungen der politischen Agenda auf „advocacy coalitions“. Ganz unterschiedliche Akteur*innen können aufgrund geteilter Überzeugungen bezüglich eines bestimmten Problems in diesen Koalitionen zusammenkommen. Die geteilten Überzeugungen müssen nicht unbedingt das Kernanliegen aller Beteiligten sein. Vielmehr koaliert eine Person auch zu eher peripheren Anliegen, vorausgesetzt die Koalition verspricht ihre Ziele voranzubringen. Die Bündelung verschiedener Ressourcen resultiert in größerer Aufmerksamkeit seitens der Entscheidungsträger*innen und erleichtert der advocacy coalition Zugang zu Entscheidungsprozessen. Die Koalition verbessert ihre Aussicht, die Agenda zu beeinflussen, durch „venue shopping “ (Baumgartner und Jones 2009 [1993]: 36-38): Das heißt, durch das Aufsuchen und Nutzen vieler verschiedener Arenen (venues) werden die Chancen erhöht, das Thema durchzusetzen (Weible und Sabatier 2007: 129; Birkland 2007: 69). Ausgelöst werden die Prozesse des Politikwandels entweder durch Policy-orientiertes Lernen jenseits der kaum veränderbaren Grundüberzeugungen (bspw. in Bezug auf instrumentelle Fragen) oder durch systemexterne Ereignisse (bspw. Regierungswechsel, Wandel der öffentlichen Meinung, technologischer Fortschritt) (Sabatier und Jenkins-Smith 1993).

Angesichts der oben vorherrschenden Fokussierung auf Themen, die an politischer Aufmerksamkeit gewinnen, gilt es zu betonen, dass auch die Erklärung des Verhinderns oder Ignorierens eines Themas ein politischer Akt sein kann, dessen Analyse einen wichtigen Baustein im Verständnis von politischem Wandel darstellt (Blum und Schubert 2018: 171). Hier legen Baumgartner und Jones (2009 [1993]) wertvolle Erkenntnisse vor, indem sie im Rahmen ihres Punctuated Equilibrium-Modells auch die Phasen politischer Stabilität ins Auge fassen. Demnach versucht jede Interessengruppe und jeder policy entrepreneur, ein Politikmonopol (,policy monopoly“) zu etablieren, das nicht nur die Deutungshoheit über das verhandelte politische Problem (,policy image“) und die Politikansätze umfasst, sondern auch die institutionellen Arrangements, die diese Deutungshoheit stützen (Baumgartner und Jones 2009 [1993]: 6). In Politikmonopolen besteht eine Konstellation aus Entscheidungsträger*innen und Interessengruppen, die sich bezüglich der Problemdeutung einig sind und die versuchen, rivalisierende Deutungen zu unterdrücken (Birkland 2007: 67-68; Jann und Wegrich 2007: 47).

Solange das policy image gesellschaftlich anerkannt oder weitgehend unhinterfragt und dementsprechend unsichtbar für die Öffentlichkeit ist, kann das Politikmonopol ungestört agieren (Birkland 2007: 67-68). Wird es zunehmend durch rivalisierende Gruppen angefochten und verliert das Politikmonopol die Kontrolle über die Agenda, werden mehr und mehr Akteur*innen aktiviert, die die 
öffentliche Aufmerksamkeit auf ihre alternativen Deutungen ziehen können (Jann und Wegrich 2007: 47). Der Politikwandel kann dann in Form relativ plötzlicher und schneller politischer Disruptionen erfolgen. In diesem Punkt, also der ,alarmed discovery stage“ bzw. dem "policy window“, verknüpfen Baumgartner und Jones (2009 [1993]: 87-88) Downs' issue-attention cycle mit Kingdons multiple streams-Ansatz. Eine besonders kurze, wenn auch partiell intensive, Aufmerksamkeitsspanne beobachten sie dann, wenn zu den verhandelten Politikproblemen keine passenden Lösungsansätze verfügbar sind. Sind aber kompatible Lösungen verfügbar, so kann das Thema im Zuge der erhöhten Aufmerksamkeit institutionalisiert werden, indem bspw. problembezogene Organisationen gegründet oder Gesetze geändert werden. Zwar sinkt auch in diesem Fall das Aufmerksamkeitsniveau nach der Modephase, es bleibt aber höher als davor (Baumgartner und Jones 2009 [1993]: 87-89).

\section{Schlussfolgerungen}

Die politikwissenschaftliche Agenda Setting-Tradition stellt ein umfassendes Arsenal von Konzepten zur Analyse von Thematisierungsprozessen bereit. Indem analog zu Akteur*innen und Interessengruppen in der Politikwissenschaft - auch wissenschaftliche Akteur*innen und Gemeinschaften um die Gültigkeit von und die Aufmerksamkeit für ihre Themen, Theorien und Modelle ringen, ist davon auszugehen, dass einige Konzepte übertragbar sind. Wie in den vorangegangenen Ansätzen der Medien und Kommunikationswissenschaften, wird auch in der Politikwissenschaft die Zyklizität von Themen betont. Dabei ist der Hinweis von Sabatier zu beachten, dass immer mehrere, interagierende Zyklen auf unterschiedlichen Ebenen ablaufen und nicht ein einzelner, linearer Prozess zur Erklärung des Zustandekommens einer Entscheidung angeführt werden kann. Damit einhergehend erfolgen Thematisierungsprozesse nicht in einem Vakuum, sondern sie entwickeln sich in Abhängigkeit von bereits bestehenden Entscheidungen, vorangegangenen und aktuellen Diskursen - so wie wissenschaftliche Innovationen stets vor dem Hintergrund bestehender Wissensstrukturen entstehen. Die Hervorhebung der Stabilität von Politikmonopolen, die zerfallen, sobald das eigene Deutungsmuster nicht mehr zu halten ist, erinnert an Kuhns Paradigmenwechsel. Für diese Arbeit zu berücksichtigen ist außerdem der multiple streams-Ansatz, dessen Übertragung schon deshalb nicht sehr abwegig zu sein scheint, weil dessen Invention auf die Untersuchung universitärer Entscheidungsstrukturen zurückgeht. Das Konzept der policy windows erscheint aber auch für die Analyse struktureller Kopplungen mit anderen Gesellschaftsbereichen aufschlussreich. So könnte bspw. die - von Planungswissenschaft und -praxis in ähnlichem Maße problematisierte - mangelnde Anwendung bzw. Anwendbarkeit planungswissenschaftlicher Lösungsansätze partiell dadurch erklärt 
werden, dass die Ströme der jeweiligen Problemwahrnehmung, der Entscheidungsprozesse und eben der geeigneten Problemlösungen nicht zusammenfinden. Dies kann wiederum für die wissenschaftsinterne Themenentwicklung Auswirkungen haben, wenn etwa die Planungswissenschaft als Reaktion auf plötzlich entstandene systemexterne Lösungserwartungen die Forschungskapazität in dem Bereich hochfährt. Genauso kann bei Vorlegen der entwickelten Lösungsansätze einige Jahre später die Anwendung und gesellschaftliche Resonanz gering ausfallen, weil sich die gesellschaftlichen Lösungserwartungen bzw. Relevanzkriterien verschoben haben. Hierbei erscheint das Bild der Surfer*innnen nützlich, die in ihrem Themenbereich jederzeit gut vernetzt mit Lösungsansätzen bereitstehen, sodass sie bei Eintreffen einer Aufmerksamkeitswelle mit etwas Geschick ihre Positionen unterbringen bzw. institutionalisieren können.

Open Access Dieses Kapitel wird unter der Creative Commons Namensnennung 4.0 International Lizenz (http://creativecommons.org/licenses/by/4.0/deed.de) veröffentlicht, welche die Nutzung, Vervielfältigung, Bearbeitung, Verbreitung und Wiedergabe in jeglichem Medium und Format erlaubt, sofern Sie den/die ursprünglichen Autor(en) und die Quelle ordnungsgemäß nennen, einen Link zur Creative Commons Lizenz beifügen und angeben, ob Änderungen vorgenommen wurden.

Die in diesem Kapitel enthaltenen Bilder und sonstiges Drittmaterial unterliegen ebenfalls der genannten Creative Commons Lizenz, sofern sich aus der Abbildungslegende nichts anderes ergibt. Sofern das betreffende Material nicht unter der genannten Creative Commons Lizenz steht und die betreffende Handlung nicht nach gesetzlichen Vorschriften erlaubt ist, ist für die oben aufgeführten Weiterverwendungen des Materials die Einwilligung des jeweiligen Rechteinhabers einzuholen. 